\title{
Pivoting an international experience preparation course during the COVID-19 pandemic
}

\author{
E. Schellhase*, M. Miller \\ Purdue University, USA
}

Keywords

Global Health

Cultural Humility

Virtual Learning

*Corresponding author:

elschell@purdue.edu

\begin{abstract}
Summary: This case study describes the adaptation of a small group interactive course, originally designed to prepare student pharmacists for an international Advanced Pharmacy Practice Experience (APPE), to a hybrid virtual course designed to prepare students to work across cultures and in many different professional environments. As the uncertainty of the COVID-19 pandemic impacted planned international experiences, a strategic pivot in delivery and content was necessary. The course content was changed to enhance student skills in cross cultural communication and humility, working through conflict, personal emotion regulation, and appropriately engaging with other cultures.
\end{abstract}

\section{Background and Context}

Purdue University College of Pharmacy offers international Advanced Pharmacy Practice Experiences (APPEs) in three countries (Kenya, United Kingdom [London and Wales] and Colombia) (Weatherman et al., 2019; Karwa et al., 2020). Each of these experiences offers students a unique learning opportunity that augments their United States-based APPEs. To ensure students are prepared to actively engage with their international sites, they are required to take a preparatory course in the spring term before APPEs begin (Schellhase et al., 2013). The course objectives focus on pharmaceutical care, disease state management, intercultural development and travel preparation. To help students meet these objectives, they participated in a number of country-specific activities along with team building activities, since each student travels to their international APPE in a group. Each student completes assignments designed to help them understand the role of the pharmacist, patient care responsibilities, and the healthcare system for the country they travel to for their APPE. Additionally, the course puts a heavy focus on helping students enhance their cultural communication and humility.

During the spring of 2020, the need to transition to virtual learning, along with the uncertainty of international APPEs occurring, triggered a significant pivot in the international preparatory course to ensure the content would still be applicable and useful for the students regardless of the rotation experiences they had during their final year. This case study highlights how this course was able to pivot the content and engage the students.

\section{Educational Description}

When the course transitioned to conduct classes virtually (at week 5 of 12), many adaptations were made to assist the students and also ensure the content would remain relevant regardless of where students participated in their APPEs. The course coordinators made an intentional shift in content to move away from the disease state and drug knowledge/ assignments and shifted the course to be focused on cultural communication and intercultural learning. It is known that many students participate in international APPEs to focus on their intercultural learning. The key elements of intercultural learning are self-awareness, awareness of the other, emotional regulation, and empathy. These skills are transferrable to all APPEs. Additionally, this course aimed to serve the students as they were struggling with stress related to the changes happening around them as the pandemic impacted their academic and personal plans. 
In light of the course's focus shifting and the need to provide a different level of attention to the students, the course was significantly altered. With the course focus changing to intercultural learning, the faculty needed to understand the mindset of each student and where they were on their individual cultural development journey. To do this, they were offered weekly office hours via WebEx. During these office hours, faculty were present and talked about whatever topic(s) the students wanted to address. Faculty also scheduled individual WebEx conversations with each student. During the conversation, faculty discussed students' intercultural assessments and worked to understand their emotions and needs within the new learning environment. These sessions typically lasted 20-45 minutes. Faculty used the information from these conversations to individualise the course for each student. The individualisation was observed in the reflection questions for each learning activity and faculty also worked with students to create an individualised intercultural learning plan. Faculty also recognised the need to provide students with ways of connecting around this content, so worked with them to use discussion boards and group office hour chats.

\section{Examples of course adaptations:}

- Small group discussions and discussion boards via Blackboard, WebEx office hours, individual student check-in meetings and cultural debriefs

- In-class activities and self-assessments: conflict style inventory, emotional hot buttons and learning style assessment (students shared their results via Qualtrics and these were then disseminated to the entire class for written reflection assignments and virtual discussions)

- Disease state case discussions and a book club with reflections (books focused on health equity and development of global health programmes)

This course used some tried and tested teaching activities in a unique delivery mechanism which allowed the course to still be impactful. This new delivery method also allowed the faculty to teach key skills needed for working in an international setting. The pivot outcome was a course that was focused on teaching cultural humility by remaining focused on individual student growth, meeting them where they were with this content, and helping students develop in ways that made sense for them.

\section{Outcomes and Recommendations}

Despite the students' devastation around the likelihood of not traveling for their planned international APPE, the course evaluations were still positive. While this wasn't exactly the same course students signed up for, they appreciated the content transition, which allowed them to learn relevant topics related to their intent for pursuing the international experience. The course received a rating of 4.58 on a 5-point scale and the overall instructor rating was a 4.83. Office hour attendance was strong. Of the 46 enrolled students, only 1 dropped this course.

\section{Student Feedback:}

'My biggest takeaway from this course is that, regardless of what happens with COVID-19, I feel prepared for all of my APPEs and I am grateful for Dr. Miller and Dr. Schellhase being open with communication and understanding of my stress.'

'The assignments made me think and will be useful in a variety of settings moving forward.'

I learned ways to prepare to interact with people from different cultures'

I learned how to better assess my personal cultural and interpersonal styles, and how these may influence my interactions with others.'

For courses preparing students for an international experience, virtual learning provides opportunities for students to gain needed knowledge while providing opportunities for individualised content and group connection. Even if students aren't able to go abroad, there is much to learn while preparing for an international experience that is applicable to domestic experiences. Lastly, asynchronous virtual learning could be used to connect students with international partners as the time zone barriers are lessened.

The adapted course met its objective of allowing students to be prepared for an international APPE while also providing them with broadly applicable knowledge and skills. The course adaptation also allowed self-discovery that can be utilised in any APPE and/or professional setting.

\section{References}

Karwa, R., Miller, M., Schellhase, E., Tran, D., Imran, M., Njuguna, B., Fletcher, S., Kanyi, J., Maina, M., Jakait, B., Kigen, G., Kipyegon, V., Arusa W., Crowe, S., \& Pastakia, S.D. (2020) Evaluating the impact of a 15-year academic partnership to promote sustainable engagement, education, and scholarship in global health. Journal of the American College of Clinical Pharmacy, 1- 12. https://doi.org/10.1002/jac5.1249

Schellhase E., Miller M., Ogallo W., \& Pastakia S. (2013). Pharmaceutical Care in Kenya: An Elective Course. American Journal of Pharmaceutical Education, 77(3), 63-67. https://doi.org/10.5688/ ajpe77360

Weatherman K., Schellhase E., Miller M., \& Al-Emran F. (2019). Assessment of intercultural learning within an advanced pharmacy practice experience in London. 79th World Congress of Pharmacy and Pharmaceutical Sciences. Abu Dhabi, United Arab Emirates. Available at: https://www.fip.org/abstracts?page=abstracts\&action=item\&item $=21436$ 